\title{
Transfer of persistence across reinforced behaviors
}

\author{
ROBERT EISENBERGER \\ University of Delaware, Newark, Delaware 19711 \\ ROBERT TERBORG \\ Calvin College, Grand Rapids, Michigan 49506
}

and

JEFFREY CARLSON

State University of New York at Albany, Albany, New York 12203

\begin{abstract}
Rats barpressed for food on a variable-interval schedule and then received food in a runway for one of three degrees of effort. Finally, all animals again barpressed for food. Requiring five runway shuttles per food pellet produced a greater subsequent rate of barpressing than reward for each shuttle, which, in turn, yielded more barpressing than free food. A follow-up study showed the five-shuttle treatment to produce more subsequent barpressing than a control condition which omitted any runway treatment. Another experiment indicated that the higher rate of barpressing following the five-shuttle treatment was not due to greater conditioned general activity, since the five-shuttle treatment failed to increase the number of grid crossings to the cue of food presentation and produced no more unconditioned barpressing than following free food in the runway. Two possible interpretations of the results were compared: (1) The degree of accustomed effort per reinforcer becomes a generalized component of instrumental behavior, (2) high effort increases the habituation of frustrationproduced disruptive responses.
\end{abstract}

A substantial amount of information exists concerning the effect of required effort upon the output of the response that is reinforced (Allison, Miller, \& Wozney, in press; Logan, 1964). However, research has only recently begun on the transfer of effort across behaviors. Lewis (cited in Spear \& Pavlik, 1966) and Wenrich, Eckman, Moore, and Houston (1967) reported that requiring rats to perform several runway traversals per food pellet produced greater subsequent performance in extinction of a second behavior, continuously reinforced barpressing, than reward for each traversal. Rashotte (1971), however, failed to replicate the Wenrich et al. results. McCuller, Wong, and Amsel (1976) found that speed during extinction of continuously rewarded runway traversal was an increasing function of the previously required number of barpresses per food pellet.

Current learning theories are unclear about such effects. According to Amsel $(1958,1972)$, the higher of two ratio requirements should result in the conditioning of the instrumental response to a greater magnitude of frustration and, therefore, produce the

Reprint requests should be addressed to Robert Eisenberger, Psychology Department, University of Delaware, Newark, Delaware 19711. higher performance of that response during extinction. However, since barpressing and runway traversal are topographically different, it is unclear from Amsel's theory why the ratio requirement for the one behavior would affect the subsequent performance of the other behavior. Amsel (1972, pp. 422-423) alluded to the possibility of a "frustration tolerance" interpretation of the partial-reinforcement extinction effect. Continued partial reinforcement would habituate the frustration-produced responses that disrupt a variety of instrumental behaviors. Therefore, the partial reinforcement of one behavior would increase the extinction performance of a topographically different behavior.

On the other hand, the organism might learn directly about the effortfulness of the instrumental process. The force applied by a rat to a lever adjusts toward the minimal amount required (Notterman \& Mintz, 1965). Lewis (1964) has shown that requiring rats to pull heavy weights for food increases the subsequent speed of running to food. And, in choice situations, there is usually a preference for the lesser required number of responses per reinforcer. Perhaps, therefore, the degree of effort expended in the successful performance of an instrumental act becomes a learned component of instrumental behavior. Then, learned effort might generalize across 
topographically different behaviors. The effort expended in one instrumental behavior would be affected both by the terms of the current instrumental contingency and the generalized effort from other reinforced behaviors. According to the learned-effort interpretation, reinforcement of one behavior would increase the effort expended in other behaviors whose reinforcement terms produced lesser effort, and would reduce the effort expended in other behaviors whose reinforcement terms produced greater effort. The greater of two ratio requirements for one behavior would be predicted to produce the more effortful performance of the second behavior. As in Logan's theory $(1956,1960)$, the learned-effort interpretation would assume that animals learn which dimensions of an instrumental behavior are monotonically related to the rapidity, quantity, or quality of reward in a particular instrumental contingency. Effort transfer would be predicted to affect performance on those dimensions of the test behavior which are, or were previously, monotonically related to reward rapidity, quantity, or quality.

The present research examined the possibility that the transfer of heightened persistence across topographically different behaviors might not be restricted to extinction, but extend to the reinforced performance of the second behavior. Food-reinforced operant barpressing by rats was tested following different numbers of required runs per reward in a runway. A variable-interval reinforcement schedule was used for barpressing to produce an intermediate base level of performance against which might be assessed the effects of different conditions of required effort in the runway. Since the test behavior was reinforced on a variable-interval schedule, transfer of persistence would be indicated by differences across groups in response rate.

\section{EXPERIMENT 1}

Rats were first trained to barpress and then given a free-operant runway task which involved one of three degrees of instrumental effort. The fixed-ratio group underwent the greatest effort, making five round trips across the length of the runway for each food pellet. Less effort was required for the continuous reinforcement group, which received a pellet for each round trip. The magazine group experienced the least effort since a pellet was delivered each time a yoked animal in the fixed-ratio group met the instrumental requirement. Following runway training, all rats again barpressed for food.

\section{Method}

Subjects and Apparatus. The subjects were 42 male SpragueDawley rats, obtained from the Holtzman Co., Madison, Wisconsin. They were about 100 days old at the start of the experiment. They were housed individually under constant illumination. Beginning 1 week prior to the start of the experiment, the rats received $14 \mathrm{~g}$ of food per day, including that consumed during the experimental session. Barpress training and testing were conducted in two identical conditioning chambers whose interior dimensions were $16.25 \times 27.94 \times 27.94 \mathrm{~cm}$. Each conditioning chamber was housed in a wooden sound-attenuated box equipped with a $15-\mathrm{W}$ fluorescent light for illumination and a fan for ventilation. The runway apparatus consisted of four similar $124 \times 9.1 \times 18.5 \mathrm{~cm}$ straight alleys. The floor and walls of the alleys were constructed of wood, while the roof was rippled Plexiglas. Photocells, used to measure shuttling, were located 25 and $96 \mathrm{~cm}$ from the food cup and were $1.25 \mathrm{~cm}$ above the floor. Experimental events were controlled and recorded automatically by solid-state programming equipment.

Procedure. The fixed-ratio, continuous-reinforcement, and magazine groups each contained 14 rats. After 1 day of gentling, each subject received 20 min of habituation to the conditioning chamber with three $45-\mathrm{mg}$ food pellets present in the food hopper. One animal that failed to consume all three pellets during the session was eliminated from the experiment. On the same day, each subject received a $20-\mathrm{min}$ period of habituation to the runway with the photocell lights on but the control devices inactive. On Day 3, each animal was magazine trained with a total of 75 pellets. On Day 4, all subjects were shaped to barpress, with the session continuing on a continuous reinforcement schedule until 75 food pellets had been delivered, including those obtained during shaping. On each of the next 3 days, each subject was reinforced for barpressing on a variable-interval 3-min (VI 3-min) schedule. Each session lasted $25 \mathrm{~min}$. The subjects were then assigned to one of the three experimental groups on the basis of matched barpress rates on the last day of training. On Day 8 , each animal was placed in the center of the runway with three pellets in the food cup. During the next 50 -min free-operant period, fixed-ratio rats received a pellet for each round trip, which consisted of traversing the alley to a point at least $96 \mathrm{~cm}$ from the food cup and returning to a point $25 \mathrm{~cm}$ from the food cup. On this day and on all subsequent days: (a) each delivery of a pellet to a fixed-ratio rat was accompanied at exactly the same moment by a pellet delivery to its paired magazine rat in an identical runway, and (b) each rat in the continuous reinforcement group remained in the alley until it earned, at a rate of one pellet per round trip, exactly the same number of pellets as its paired fixed-ratio rat. On Day 9, the fixed-ratio group received one pellet for every completion of two round trips, every three trips on Day 10, every four trips on Day 11, and every five trips on Days 12-14.

One day following the last day of runway training, each subject was returned to the conditioning chamber for a 60 -min session with barpresses reinforced on the VI 3-min schedule.

\section{Results}

In training, the mean numbers of barpresses per minute for the 3 days on the VI schedule were, respectively, $6.64,9.24$, and 10.8 . The three groups did not differ significantly on the last day of training ( $F<1.0)$.

The test results were as predicted. The fixed-ratio group had a much greater rate of barpressing than the continuous reinforcement group, which, in turn, outperformed the magazine group. Each subject's proportional change of barpress performance from training to test was calculated as follows. The number of responses in each of two 25 -min test blocks was divided by the number of responses during the last 25-min training session. The average ratios for each group are given in Figure 1, while the average barpress 


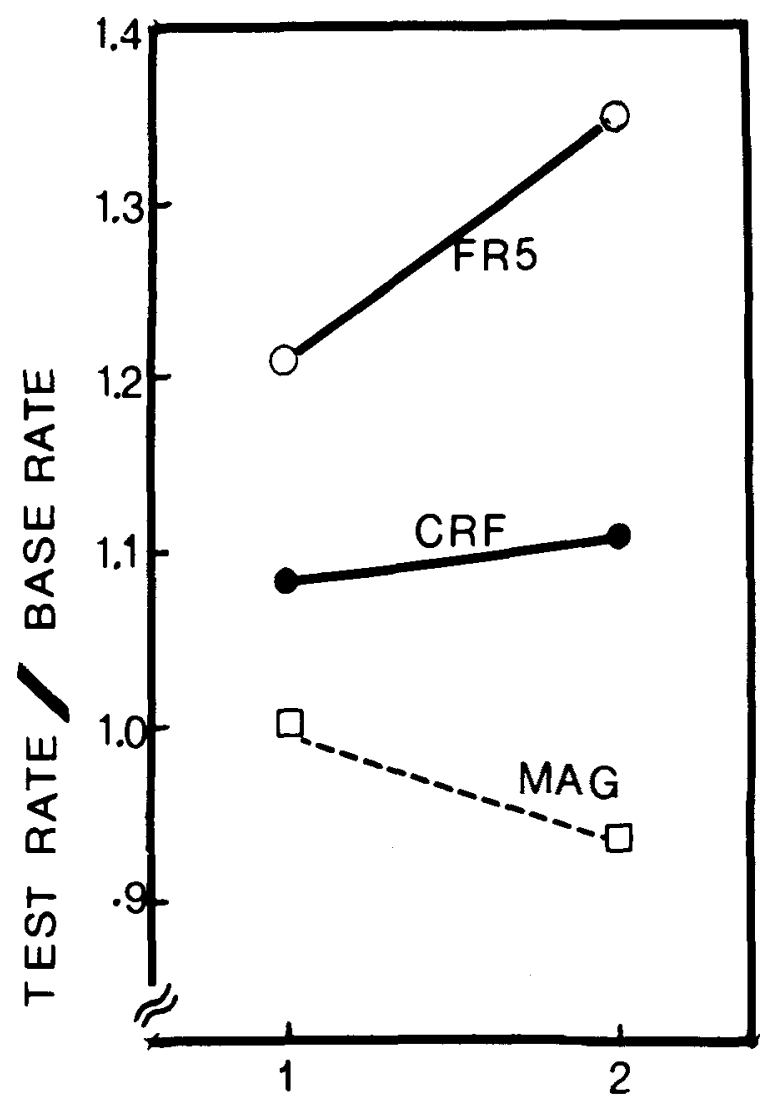

BLOCKS OF 25 MINUTES

Figure 1. The mean ratio of barpresses in test to barpresses in training for the fixed-ratio, continuous-reinforcement, and magazine groups (Experiment 1).

rates in the last training session and the two test periods are given for each group in Table 1. Inclusion of the last $10 \mathrm{~min}$ of test data, omitted in order to perform the statistical analysis, would not affect conclusions concerning statistical significance. Experimental condition was treated as a matchedsubject factor because subjects were assigned to groups on the basis of matched barpress rates in training. A $3 \times 2$ (Group by Trial Block) repeated measures analysis of variance on the two $25-\mathrm{min}$ test periods revealed a significant effect only for groups $[F(2,65)=8.31, p<.01]$. The fixed-ratio group had a greater rate of barpressing than the continuous reinforcement group, which, in turn,

Table 1

Average Number of Barpresses per Minute in the Final Training Session and the Two Test Periods

\begin{tabular}{lcccc}
\hline \multicolumn{1}{c}{ Group } & \begin{tabular}{c} 
Training \\
\cline { 4 - 5 } Session 3
\end{tabular} & & \multicolumn{2}{c}{ Test Period } \\
\hline Fixed Ratio & 11.0 & 12.7 & 14.0 \\
Continuous Reinforcement & 10.7 & 11.1 & 11.4 \\
Magazine & 10.8 & 10.5 & 10.0 \\
\hline
\end{tabular}

outperformed the magazine group [respective $\mathrm{ts}(65)$ $=2.39,1.67$, ps $<.02, .10]$.

These results are not attributable to differential weight loss across groups since the posttest weights of the fixed-ratio, continuous-reinforcement, and magazine groups (respectively 254,247 , and $252 \mathrm{~g}$ ) did not differ systematically $(\mathrm{F}<1.0)$.

To determine whether the runway treatment increased the barpress performance of the fixed-ratio rats, as opposed to decreasing the performance of the remaining groups, a subsequent experiment compared the performance of a fixed-ratio group $(n=11)$ with a control group $(\mathrm{n}=11)$ that was trained to barpress but given no runway treatment during the 8-day interval between training and test. The fixed ratio group's average increase in barpressing above the baseline performance, $35 \%$, was similar in magnitude to the first experiment, while the control group decreased $5 \%$ below the baseline. The difference between the groups was significant $[\mathrm{t}(20)=2.77$, $\mathrm{p}<.02]$. In summary, (a) the rate of operant barpressing was an increasing function of the required effort in the runway, and (b) the fixed-ratio treatment increased the rate of barpressing above that of a control group not receiving the runway treatment.

\section{EXPERIMENT 2}

This experiment tested the possibility that the results of the first experiment were due not to the transfer of reinforced persistence, but to differences of general activity, conditioned to the cue of food presentation, which could have altered the unconditioned probability of barpressing. In order to assess the effects of food presentation upon general activity and upon unconditioned barpressing, all rats received intermittent free presentations of food in the conditioning chamber both before and after receiving the fixed-ratio treatment or magazine treatment in the runway. The conditioned activity interpretation provided three predictions: (a) prior to the runway treatment, there would be a positive correlation between the rates of general activity and unconditioned barpressing; (b) fixed-ratio reinforcement of runway traversal would result in greater subsequent general activity in the conditioning chamber to the cue of food presentation as compared to the magazine condition; and (c) fixed-ratio reinforcement of runway traversal would produce a greater rate of unconditioned barpressing in the conditioning chamber to the cue of food presentation as compared to the magazine condition.

\section{Method}

The subjects were 16 male 100-day-old Sprague-Dawley rats, obtained from the same source as in the preceding experiment. The apparatus was the same as in Experiment 1. In order to 
measure general activity in the conditioning chamber, a clear Plexiglas floor was employed whose bottom surface was divided into a four-quadrant grid by a pair of perpendicular narrow strips of black tape. Each movement of the rat's nose across a tape boundary was tallied as a grid crossing by a carefully trained observer who viewed the rat through a one-way mirror located in the top of the soundproof enclosure. The observer was unaware of the animal's group. During training and test in the conditioning chamber, the rats received the same number and average temporal distribution of food presentations per day as in the prior study, with deliveries now independent of behavior. On Days 1-3, all rats received precisely the same gentling, habituation, and magazine-training procedures as in Experiment 1. On Day 4, the rats received the same number and average temporal distribution of food presentations in the conditioning chamber as was received by the rats in the previous experiment during the course of shaping. On Days 5-7, all rats received food presentations in the conditioning chamber independently of their behavior on a variable schedule averaging once every three minutes (VT $3 \mathrm{~min}$ ). Half the animals were then assigned to the fixed-ratio group, and the remainder to the magazine group. Runway training for these groups was conducted exactly as in the first experiment. Next, all animals were returned to the conditioning chamber for a $60-\mathrm{min}$ period in which food was again presented on the VT 3-min schedule, and measures of general activity and unconditioned barpressing were obtained. The use in training of food presentations in the conditioning chamber assured that, in test, the animals would approach and eat the reward pellets immediately following each presentation so that differences across groups in the possible cue effects of the pellets upon activity could be assessed.

\section{Results}

Contrary to the conditioned activity interpretation, on the last day in the conditioning chamber prior to the runway treatment there was only a very small, statistically nonsignificant, Spearman rank correlation between the numbers of grid crossings and barpresses $\left(\mathrm{r}_{\mathrm{s}}=.15, \mathrm{t}(14)=.58\right)$. This result indicates that high general activity had a negligible effect on the unconditioned rate of barpressing.

During runway training, one fixed-ratio rat developed a respiratory illness and was discarded along with his yoked magazine rat. As illustrated in Figure 2, following runway training the fixed-ratio group made about the same number of grid crossings as before and the magazine group made a nonsignificantly greater number of grid crossings than the fixed-ratio group. The average rates of grid crossing for each grolip in the last training session and in the test session are given in Table 2. Each subject's proportional change from training to test in grid crossings was calculated in the same way as the barpress data of the preceding experiment. A $2 \times 2$ (Group by Trial Block) repeated measures analysis of variance revealed a significant effect for trial blocks $[\mathrm{F}(1,12)=7.82, \mathrm{p}<.05]$, and only an insignificant effect for groups $[\mathrm{F}(1,12)=2.71$, $p>.10]$. Therefore, the superior performance of the fixed-ratio group in the first experiment is not attributable to an increase in general activity, conditioned to food presentations.

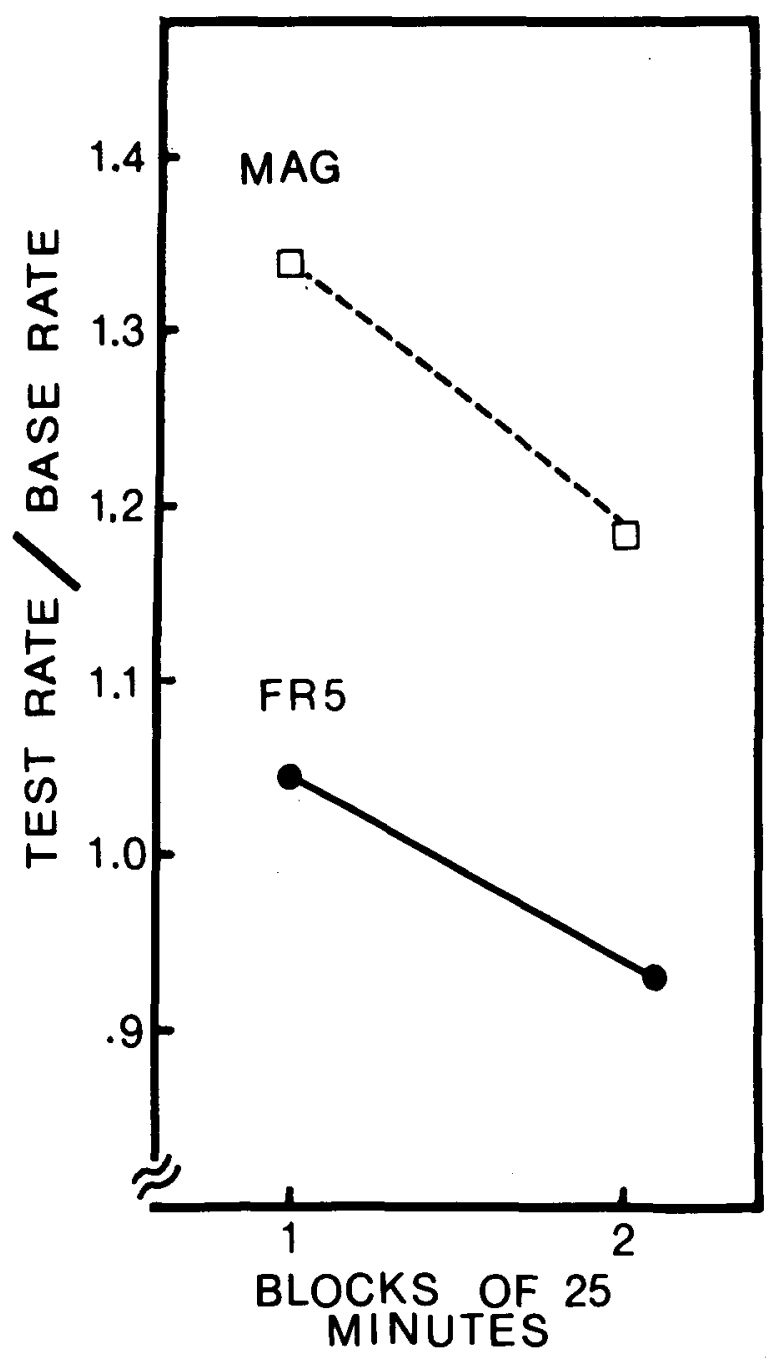

Figure 2. The mean ratio of grid crossings in test to grid crossings in training for the fixed-ratio group and magazine group (Experiment 2).

On the measure of unconditioned barpressing, the two groups deviated markedly from normality so that a nonparametric statistical analysis was employed. For both groups, the median number of barpresses on the last day in the conditioning chamber prior to the runway treatment was 13 . The median number of barpresses in test (which, it will be recalled, lasted $60 \mathrm{~min}$ or 2.4 times the duration of the last VT training session) was 32 for the fixedratio group and 28 for the magazine group. Since,

Table 2

Average Number of Grid Crossings per Minute in the Final Training Session and the Two Test Periods

\begin{tabular}{lccc}
\hline & Training & \multicolumn{2}{c}{ Test Period } \\
\cline { 3 - 4 } \multicolumn{1}{c}{ Group } & Session 3 & 1 & 2 \\
\hline Fixed Ratio & 12.0 & 12.2 & 10.6 \\
Magazine & 11.4 & 14.9 & 13.0 \\
\hline
\end{tabular}


as previously noted, the distributions of the number of barpresses in training deviated markedly from normality and since one subject had a zero barpress rate prior to the runway treatment, rather than computing a training/test ratio for each rat, a difference score was calculated by subtracting the rat's rate following the runway treatment from the rate before the runway treatment. The difference between the two groups did not approach statistical significance $(U=18, p=.46$, Mann-Whitney $\mathrm{U}$ test). Thus, there is no evidence that the incremental effect of the fixed-ratio runway treatment was the result of an increase in the unconditioned barpress rate.

In interpreting the present results, it should be cautioned that the measure of general activity was only one of a number of possible alternatives. Moreover, accepting the null hypothesis does not necessarily indicate that the degree of reinforced effort in the runway had no effect upon the rate of unconditioned barpressing. The results do indicate that any effect of the runway manipulation upon the rate of unconditioned barpressing was of very small magnitude and, therefore, unlikely to account for the results of the first experiment.

\section{DISCUSSION}

In the first experiment, rats required to make five runway shuttles per food pellet had a greater subsequent rate of barpressing than rats rewarded for each shuttle who, in turn, outperformed rats that received free food. The runway treatment increased the fixed-ratio group's rate of barpressing, as opposed to merely decreasing the performance of the remaining groups, since a follow-up study found that the fixedratio group outperformed a control group that was trained to barpress but received no treatment during the interval between training and test. The second experiment indicated that superior performance of the fixed-ratio group was not due to increased general activity, conditioned to the cue of food presentation, which might have affected the unconditioned probability of barpressing. Contrary to the conditioned-activity interpretation, (a) prior to the runway treatment, there was only a negligible correlation between the numbers of grid crossings and barpresses; (b) following the fixed-ratio treatment, the number of grid crossings did not increase and was nonsignificantly less than after the magazine treatment; and (c) the unconditioned barpress rates produced by the fixed-ratio and magazine treatments were highly similar.

Since barpressing is topographically different from runway shuttling, the results cannot be attributed to primary response generalization. The learned effort interpretation of the present results would assume that if tasks which customarily produced the soughtafter reinforcer were currently unavailable or ineffective, stimuli that evoked the relevant task behavior would affect effort in other behaviors which are, or were previously, correlated with reinforcement. Such cues might include the intermittent presentation of the reinforcer itself, discriminative stimuli, and the general environmental context. Note that accustomed effort would not be assumed to transfer indiscriminately across stimulus settings. The more different the transfer situation from the setting where high effort was reinforced, the weaker would be the transfer effect. It also follows that it should be possible to bring generalized effort under discriminative control. Following discrimination training in which the discriminanda signaled different fixed ratios, these discriminanda should cue differential effort in the performance of another reinforced behavior.

The alternative, frustration-tolerance interpretation might be extended to the present results by assuming that the disruptive effects of frustration on an intermittent reinforcement schedule may be reduced by prior habituation on an intermittent reinforcement schedule for a different behavior. The frustration tolerance mechanisms implies a more global persistence characteristic than learned effort because transfer of persistence across tasks would not require common cues, merely that both tasks involved sufficient frustration. Also, the frustration-tolerance interpretation would assume that individuals can only increase, never decrease, the generalized ability to cope with frustration, whereas the learned effort mechanism predicts that generalized persistence may be reduced by repeated experience with low-effort reward. The distinctive predictions of these two interpretations remain to be tested.

It is, of course, possible that both the learnedeffort and frustration mechanisms contribute to general persistence, in which case general decremental effects of rewarded low effort would occur but be weaker in magnitude than the general incremental effects of rewarded high effort. The reason is that both mechanisms would act to increase general persistence following reward for high effort, whereas only one mechanism would act to decrease general persistence following rewarded low effort. Another possible interpretation is based upon Lawrence and Festinger's (1962) suggestion that high effort increases the reward value of consequent stimulation. It might be argued that the fixed-ratio shuttle requirement increased the reward value of the food reward. Contrary to this interpretation, however, Mirsky (1975) failed to find a preference for a goalbox that previously had been paired with a stringent 
instrumental requirement vs. a distinctively different goalbox that previously had been paired with a lesser instrumental requirement.

The present results extend to reinforced performance the previous findings that the higher of two ratio requirements for one instrumental behavior increased the subsequent extinction performance of a second, topographically different behavior. The learned-effort view also suggests that varying effort in ways other than the required number of responses, such as response force, would produce similar results. Moreover, a fine-grain analysis of transfer performance should indicate generalized effort in all those quantitative dimensions which are monotonically related to reward rapidity, quantity, or quality. For example, on free-operant schedules in which performing the response takes appreciable time, both the interval between responses and the speed of the response itself should be affected.

\section{REFERENCES}

Allison, J., Miller, M., \& Wozny, M. Conservation in behavior. Journal of Experimental Psychology: General, in press.

AMSEL, A. The role of frustrative nonreward in noncontinuous reward situations. Psychological Bulletin, 1958, 55, 102-119.

Amsel, A. Behavioral habituation, counterconditioning, and a general theory of persistence. In A. Black \& W. Prokasy (Eds.),
Classical conditioning II: Current theory and research. New York: Appleton-Century-Crofts, 1972.

Lawrence, D. H., \& Festinger, L. Deterrents and reinforcement. Stanford: Stanford University Press, 1962.

LEwis, M. Some nondecremental effects of effort. Journal of Comparative and Physiological Psychology, 1964, 57, 367-372.

LogAN, F. A. A micromolar approach to behavior theory. Psychological Review, 1956, 63, 63-73.

Logan, F. A. Incentive. New Haven, Conn: Yale University Press, 1960.

Logan, F. A. The free behavior situation. In D. Levine (Ed.), Nebraska Symposium on Motivation. Lincoln: University of Nebraska Press, 1964.

McCuller, R., Wong, P. T. P., \& Amse L, A. Transfer of persistence from fixed-ratio barpress training to runway extinction. Animal Learning \& Behavior, 1976, 4, 53-57.

Mirsky, M. Effects of instrumental effort and food deprivation on the development of incentive motivation. Unpublished doctoral dissertation, State University of New York at Albany, 1975.

Notterman, J. M., \& Mintz, D. E. Dynamics of response. New York: Wiley, 1965.

RASHotTE, M. E. Influence of partial reinforcement of running on the extinction of continuously reinforced barpressing in rats. Psychonomic Science, 1971, 25, 145-147.

Spear, N. E., \& Pavlik, W. B. Percentage of reinforcement and reward magnitude effects in a $\mathrm{T}$ maze: Between and within subjects. Journal of Experimental Psychology, 1966, 71, 521-528.

Wenrich, W. W., Eckman, G. E., Moore, J. J., \& Houston, D. F. A trans-response effect of partial reinforcement. Psychonomic Science, 1967, 9, 247-248.

(Received for publication October 6, 1978; revision accepted March 2, 1979.) 